\title{
Pyrrolidine Dithiocarbamate Attenuates Paraquat-Induced Lung Injury in Rats
}

\author{
Xiuli Chang, Chunfeng Shao, Qing Wu, Qiangen Wu, Min Huang, and Zhijun Zhou \\ Department of Occupational Health and Toxicology, School of Public Health, Fudan University, Shanghai 200032, China \\ Correspondence should be addressed to Zhijun Zhou, zjzhou@shmu.edu.cn
}

Received 27 February 2009; Accepted 26 May 2009

Recommended by Michael Cunningham

\begin{abstract}
Paraquat (PQ) has been demonstrated that the main target organ for the toxicity is the lung. This study aimed to investigate the potential protective effect of PDTC on the PQ-induced pulmonary damage. Fifty-four rats were divided into control, PQ-treated and PQ+PDTC-treated groups. Rats in the PQ group were administrated $40 \mathrm{mg} / \mathrm{kg}$ PQ by gastric gavage, and PDTC group with $40 \mathrm{mg} / \mathrm{kg}$ PQ followed by injection of $120 \mathrm{mg} / \mathrm{kg}$ PDTC (IP). On the days 3, 7, 14 and 21 after treatments, the activities of GSH-Px, SOD, MDA level and the content of HYP were measured. TGF- $\beta 1$ mRNA and protein were assayed by RT-PCR and ELISA. MDA level in plasma and BALF was increased and the activities of GSH-Px and SOD were decreased significantly in the PQ-treated groups $(P<.05)$ compared with control group. While the activities of GSH-Px and SOD in the PQ+PDTC-treated groups was markedly higher than that of PQ-treated groups $(P<.05)$, and in contrast, MDA level was lower. TGF- $\beta 1$ mRNA and protein were significantly lower in the PQ+PDTC-treated groups than that of $\mathrm{PQ}$-treated groups $(P<.05)$. The histopathological changes in the PQ+PDTC-treated groups were milder than those of PQ groups. Our results suggested that PDTC treatment significantly attenuated paraquat-induced pulmonary damage.
\end{abstract}

Copyright () 2009 Xiuli Chang et al. This is an open access article distributed under the Creative Commons Attribution License, which permits unrestricted use, distribution, and reproduction in any medium, provided the original work is properly cited.

\section{Introduction}

Paraquat $(\mathrm{PQ})$ is a nonselective contact herbicide, which is used world-widely for its high efficiency and low residues in the crops. It has been extensively demonstrated that it is highly toxic to multiorgans when absorbed through ingestion, skin contact, or inhalation. The primary target organ for PQ toxicity is the lung as a consequence of its accumulation, against a concentration gradient, through the highly developed polyamine uptake system [1-3]. The toxicity mechanism of PQ is mainly due to a sustained redoxcycling effect, resulting in oxidative stress-related insults such as lipid peroxidation. PQ-induced lung injury results in alveolar epithelial cells (type I and II pneumocytes) and Clara cell disruption, impairments of the surfactant system, hemorrhage, edema, hypoxemia, infiltration of inflammatory cells into the interstitial and alveolar spaces, proliferation of fibroblasts, and excessive collagen deposition [3, 4], which ultimately leads to pulmonary fibrosis and respiratory failure. Survivors of PQ poisoning may be left with a restrictive type of long-term pulmonary dysfunction [5]. So far, there are no known pharmacological antidotes for PQ poisoning [6-8] and therapeutics have been disappointing and the mortality has still remained high [9]. Thus agent with both antioxidant and regulating fibrosis properties would have favorable value in the treatment of paraquat-induced lung injury.

Pyrrolidine dithiocarbamate (PDTC) is a low-molecularweight thiol compound, which has a variety of biochemical activities, such as redox state alternation $[10,11]$, heavy metal chelation [12], and enzyme inhibition [13]. PDTC was initially regarded as a potent inhibitor of NF- $\kappa \mathrm{B}[10$, 14], and it has been used as an antioxidant compound to counteract the toxic effects of free radicals [15] and to interfere with the generation of proinflammatory cytokines [16]. It has the potential to activate gene expression of endogenous antioxidants such as superoxide dismutase [17], independent of any effects on NF- $\kappa \mathrm{B}$. It induces the genes encoding the two subunits of the enzyme GCS and increased de novo synthesis of the cellular protectant GSH [18]. PDTC reduces oxidant-mediated cellular injury, as demonstrated by a reduction in the accumulation of 
malondialdehyde [19]. Antioxidants such as PDTC and their modulatory effects on NF- $\kappa \mathrm{B}$ activation suggest that these agents may offer therapeutic benefits in acute lung injury caused by PQ. Therefore, the present study was designed to evaluate the effects of PDTC in a rodent model of acute lung injury induced by PQ and observe its potential therapeutic effect in order to provide scientific basis for the treatment strategy of paraquat-induced lung damage.

\section{Materials and Methods}

2.1. Chemicals. Pyrrolidine dithiocarbamate ( $\geq 99 \%)$ was purchased from Sigma-Aldrich (St. Louis, Mo, USA). 45\% paraquat concentration was gifted by Sinon Chemical (Shanghai) Co., Ltd. All other chemicals were of analytical grade, and procured from local commercial sources.

2.2. Animals and Paraquat/PDTC Administration. Fifty-four male Sprague-Dawley (SD) rats which initially weighed between 180 and $220 \mathrm{~g}$ were purchased from the Laboratory Animal Research Center of Fudan University. The animals had free access to the pellet diet and water ad libitum always, and were maintained on 12-hour diurnal cycles and in a controlled environment with a temperature of $20 \sim 22^{\circ} \mathrm{C}$ and humidity of $50 \pm 5 \%$ for a period of 1 week before usage. Animals were randomly divided into the control group, $n=$ 6: animals treated with the saline solution; the PQ group, $n=$ 24: animals were orally given aqueous solution of paraquat $(40 \mathrm{mg} / \mathrm{kg})$ by gastric gavage and sacrificed at 3rd day $(n=6$, PQ 3d group), 7 th day ( $n=6$, PQ 7 d group), 14 th day ( $n=6$, PQ 14 d group) and 21 st day $(n=6$, PQ $21 \mathrm{~d}$ group); PQ+PDTC group, $n=24$ : animals intoxicated with $40 \mathrm{mg} / \mathrm{kg}$ PQ followed by immediate injection $120 \mathrm{mg} / \mathrm{kg}$ PDTC (IP), and sacrificed at 3 rd day $(n=6, \mathrm{PQ}+\mathrm{PDTC} 3 \mathrm{~d}$ group), 7 th day $(n=6, \mathrm{PQ}+\mathrm{PDTC} 7 \mathrm{~d}$ group $), 14$ th day $(n=6$, $\mathrm{PQ}+\mathrm{PDTC} 14 \mathrm{~d}$ group), and 21st day $(n=6, \mathrm{PQ}+\mathrm{PDTC} 21 \mathrm{~d}$ group).

2.3. Tissue Preparation. On days 3, 7, 14, and 21 after treatments, one group rats of each treatment were sacrificed with an IP injection of $10 \%$ Chloralum Hydratum $(3 \mathrm{~mL} / \mathrm{kg}$ body weight). A total of $6 \mathrm{~mL}$ of venous whole blood was collected in heparin-containing Vacutainer, and centrifuged at $400 \mathrm{~g}$ for 10 minutes collecting supernatant for measurement of the activities of glutathione peroxidase (GSH-Px), superoxide dismutase (SOD), the level of maleic dialdehyde (MDA), and TGF- $\beta 1$ protein.

After collected blood sample, immediately thoracotomy was sterilely performed to obtain bronchoalveolar lavage fluid (BALF). Once ligating the left major bronchial beneath the tracheal crotch, Bronchoalveolar lavage (BAL) was performed by flushing right lung with $5 \mathrm{~mL}$ of saline through the tracheal cannula three times. About $3 \mathrm{~mL}$ BALF was pooled and centrifuged at $300 \mathrm{~g}$ for 10 minutes. The supernatant was harvested for the activities of SOD, GSH-Px, and MDA analysis.
Right lung was harvested and frozen at $-80^{\circ} \mathrm{C}$ for RNA extraction and the content of hydroxyproline (HYP) in lung homogenate measurement, a small piece of left lung was fixed in 3\% glutaraldehyde and immediately sent for ultra-morphological examination, and the remaining lungs were fixed in $10 \%$ formaldehyde solution before histological analysis. HE and Masson's trichrome staining of lung section were undertaken, the latter was used observing collagen fibers. According to the methodology described by Szapiel, results of microscopic observations were analyzed the semiquantitative [20].

2.4. Measurement of Biomarkers of Oxidative Stress. The levels of MDA were determined as an indicator of lipid peroxidation. The activities of GSH-Px, SOD, and the level of MDA both in plasma and BALF of rats were measured using qualified kits.

2.5. Hydroxyproline Assay of Lung Tissue. The hydroxyproline contents of lung tissues were determined and the data were expressed as ng/g wet lung tissue. The $100 \mathrm{mg}$ frozen lung tissue from control, PQ-treated and, PQ+PDTC-treated rats was thoroughly homogenized in distilled water and measured using qualified kits.

2.6. TGF- $\beta 1$ Gene Expressions by Reverse TranscriptionPolymerase Chain Reaction (RT-PCR). About $100 \mathrm{mg}$ lung tissue was ground into a powder in liquid nitrogen. Total RNA was extracted using the TRIzol Reagent (Life Technologies, Grand Island, NY, USA) according to the manufacturer's instructions. Yield and purity of the isolated RNA solution were determined by A260 and A280 readings on a spectrophotometer. Reverse transcription was performed on $3 \mu \mathrm{g}$ of RNA with oligo-dT primers and avian myeloblastosis virus reverse transcriptase (MBI Fermentas, St. Leon-Rot, Germany). The PCR carried out with the primers. The primers for mRNA analysis were upper 5' GCTCGCTTTGTACAACAGCA $3^{\prime}$ and lower 5' GAGTTCTACGTGTTGCTCCA 3' yielding a 280-bp product for TGF- $\beta 1$. The primers for mRNA analysis were upper $5^{\prime}$ CCTCTATGCCAACACAGTGC $3^{\prime}$ and lower 5' GTACTCCTGCTTGCTGATCC $3^{\prime}$ yielding a 210-bp product for housekeeping gene $\beta$-actin. The PCR products were analyzed by electrophoresis on an agarose gel, stained with ethidium bromide, and photographed. To determine the linear range of the PCR, the intensity of the amplified products was plotted against the cycle number. At least three samples on each day were analyzed in each group.

2.7. Measurements of TGF- $\beta 1$ Level in Plasm. The plasm sample was used for measurements TGF- $\beta 1$ levels with enzyme-linked immunosorbent assay Rat TGF- $\beta 1$ kit (Bender MedSystems, Lot: 20280013) according to the manufacturer's protocol. TGF- $\beta 1$ was expressed as ng/g of protein.

2.8. Protein Quantification. Protein quantification was performed according to the method of Lowry et al. [21], using bovine serum albumin as standard. 
TABLE 1: Comparisons of GSH-Px, SOD, and MDA among groups of PQ treatment, PQ+PDTC treatment, and the control (Mean \pm SE).

\begin{tabular}{|c|c|c|c|c|c|c|c|c|}
\hline \multirow{2}{*}{$\begin{array}{l}\text { Animal } \\
\text { group }\end{array}$} & \multirow{2}{*}{$n$} & \multirow{2}{*}{$\begin{array}{c}\text { Sacrifice } \\
\text { Time } \\
(d)\end{array}$} & \multicolumn{2}{|c|}{ GSH-Px $(\mathrm{U} / \mathrm{mL})$} & \multicolumn{2}{|c|}{$\mathrm{SOD}(\mathrm{U} / \mathrm{mL})$} & \multicolumn{2}{|c|}{ MDA (nmol/mgprot) } \\
\hline & & & Blood plasma & BALF & Blood plasma & BALF & Blood plasma & BALF \\
\hline control & 6 & 0 & $5147.60 \pm 94.34$ & $60.28 \pm 13.86$ & $288.62 \pm 2.88$ & $59.51 \pm 21.60$ & $1.09 \pm 0.22$ & $0.46 \pm 0.30$ \\
\hline PQ & 5 & 3 & $4456.57 \pm 122.10^{* *}$ & $18.99 \pm 5.20^{* *}$ & $100.95 \pm 3.74^{* *}$ & $10.12 \pm 2.72^{* *}$ & $3.27 \pm 1.51^{*}$ & $1.76 \pm 0.30 *$ \\
\hline treatment & 6 & 7 & $4194.06 \pm 154.30^{* *}$ & $11.28 \pm 2.43^{* *}$ & $99.93 \pm 0.34^{* *}$ & $12.49 \pm 10.89^{* *}$ & $3.21 \pm 0.12^{* *}$ & $0.76 \pm 0.20^{*}$ \\
\hline \multirow{6}{*}{$\begin{array}{l}\mathrm{PQ}+ \\
\text { PDTC } \\
\text { treatment }\end{array}$} & 6 & 14 & $3907.14 \pm 465.88^{* *}$ & $24.71 \pm 10.39^{* *}$ & $275.73 \pm 20.24$ & $42.11 \pm 8.80$ & $0.87 \pm 0.07$ & $0.61 \pm 0.04$ \\
\hline & 6 & 21 & $4655.24 \pm 291.08^{* *}$ & $42.67 \pm 14.94$ & $285.37 \pm 10.57$ & $70.58 \pm 5.54$ & $0.32 \pm 0.06^{* *}$ & $0.16 \pm 0.02^{*}$ \\
\hline & 5 & 3 & $4735.35 \pm 188.20 \boldsymbol{\Lambda}^{* *}$ & $19.39 \pm 4.80^{* *}$ & $102.13 \pm 2.63^{* *}$ & $18.28 \pm 6.48^{* *}$ & $2.74 \pm 0.08^{* *}$ & $0.90 \pm 0.50 \Delta \boldsymbol{\Delta} *$ \\
\hline & 6 & 7 & $4384.16 \pm 91.93 \boldsymbol{\Delta}^{* *}$ & $18.61 \pm 8.18^{* *}$ & $103.45 \pm 0.98 \boldsymbol{\Delta} \mathbf{\Delta}^{* *}$ & $15.53 \pm 5.49^{* *}$ & $2.38 \pm 0.40 \boldsymbol{\Delta} \mathbf{\Delta}^{* *}$ & $0.71 \pm 0.44$ \\
\hline & 6 & 14 & $4307.14 \pm 21.43^{* *}$ & $43.95 \pm 8.61 \mathbf{\Delta} \mathbf{\Delta}^{*}$ & $322.49 \pm 22.85 \boldsymbol{\Lambda} \mathbf{\Lambda}^{*}$ & $61.08 \pm 10.34 \boldsymbol{\Lambda} \Delta$ & $0.30 \pm 0.01 \mathbf{\Delta} \mathbf{\Delta}^{* *}$ & $0.62 \pm 0.01$ \\
\hline & 6 & 21 & $5001.90 \pm 508.37$ & $62.48 \pm 8.66 \boldsymbol{\Delta}$ & $365.09 \pm 16.17 \mathbf{\Delta} \mathbf{\Delta}^{* *}$ & $108.43 \pm 21.17 \boldsymbol{\Delta} \mathbf{\Delta}^{* *}$ & $0.32 \pm 0.03^{* *}$ & $0.08 \pm 0.03^{* *}$ \\
\hline
\end{tabular}

*Compared with the control, $P<.05,{ }^{*} * P<.01, \boldsymbol{\Delta}$ compared with the PQ treatment, $P<.05, \boldsymbol{\Delta} \mathbf{\Delta} P<.01$.

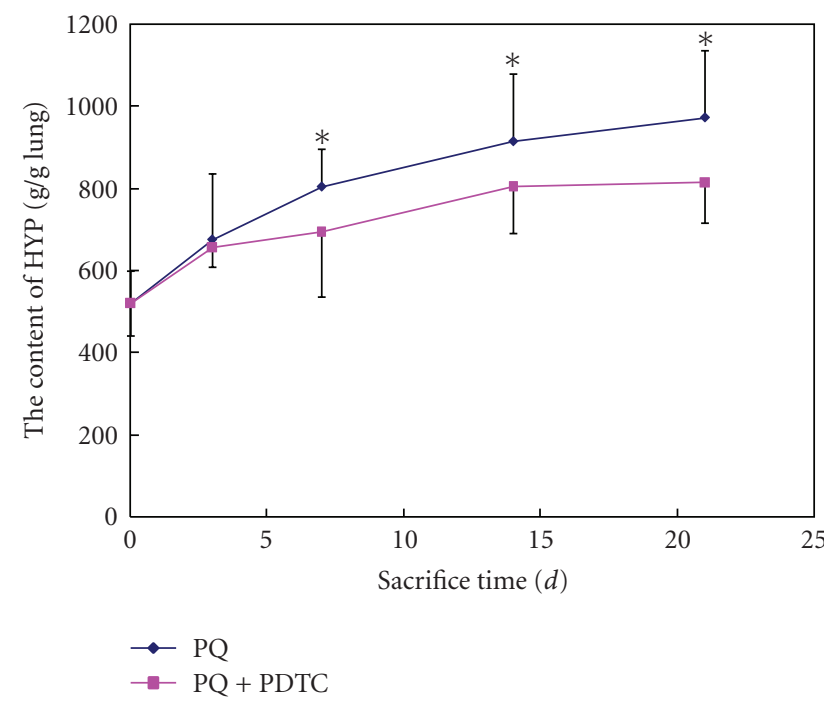

Figure 1: The content of HYP in lung tissue from groups of the control, PQ treatment, and PQ+PDTC treatment. * compared with the control, $P<.01$

2.9. Statistical Analysis. Results are presented as the mean \pm SE. We used ANOVA to determine the differences among groups. Comparisons between control, PQ-treated, and PQ+PDTC-treated groups at each time point were made using unpaired Student's $t$-test. Differences were considered significant at $P<.05$.

\section{Results}

3.1. Survival Rate and Macroscopic Examination. Polypnea, Blausucht, crouch, diarrhea, anorexia were present especially in animals exposed to PQ during the first 24 hours. One animal died on the third day after treatment in the PQ group and PQ+PDTC group, respectively. There was no animal death in other goups during the observed time.
3.2. PDTC Alleviated the PQ-Induced Oxidative Damages. The results of our experiment using $P Q$-treated rats ( $40 \mathrm{mg} / \mathrm{kg}$ PQ) indicated that lipid peroxidation marker MDA levels in plasma and BALF were significantly increased and the activities of GSH-Px and SOD were significantly decreased $(P<.01)$ compared with that in the control group. When the rats were cotreated with PQ and PDTC, we found the activities of GSH-Px and SOD in the PDTC treatment group $(120 \mathrm{mg} / \mathrm{kg})$ was markedly higher than that of PQtreated group $(P<.05$ or $P<.01)$, and the MDA levels was lower correspondingly (Table 1 ). The content of HYP in lung tissue was increased significantly $(P<.05)$ compared with the control group at 7th day after the treatment of PQ $40 \mathrm{mg} / \mathrm{kg}$, while the content of HYP in lung tissue was slightly decreased in cotreated with PQ and PDTC group (Figure 1).

3.3. PDTC Alleviated the Pathological Changes Caused by PQ as Determined by Histochemistry and Electronic Microscopy. PQ-induced lung structural, ultrastructural alterations, and alleviative effects of PDTC on PQ-damages are depicted in Figures 2 and 3. Histologic changes were assessed with $\mathrm{H} \& \mathrm{E}$ and correlated with lung fibrosis, which was identified by using Masson's Trichrome stain for collagen. According to the methodology described by Szapiel, the semiquantitative results of microscopic observations were summarized in Table 2. Animals from control group (saline solution) presented a normal pulmonary structure at light microscopy (LM) and electron microscopy (EM), without evidences of alveolar collapse, cellular infiltrations, or collagen accumulation. PQ administration induced marked alterations compared to the control pattern, mainly characterized by a diffuse alveoli collapse with an increased thickness of its walls. It was noticed that an intense vascular congestion with numerous activated platelets and polymorphonuclear cells inside the capillaries. The majority of pneumocytes showed, at least, one ultrastructural abnormality, mitochondrial swelling being the most frequent alteration, abundant rough endoplasmic reticulum (RER), and rich ribosome in the fibroblast. In comparison with the PQ group, the occurrence 


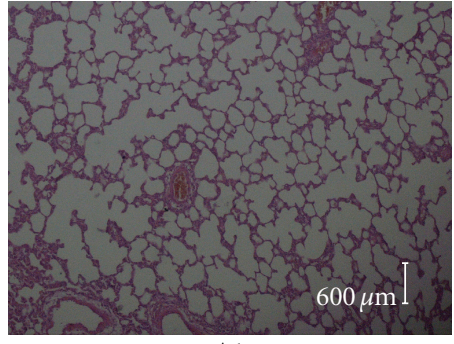

Al

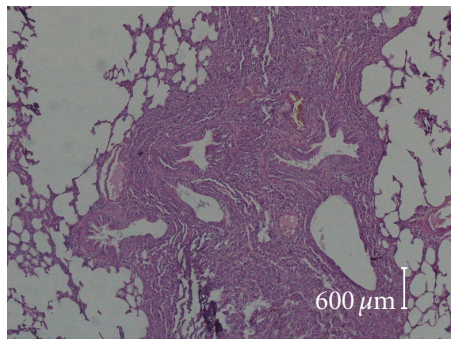

B1

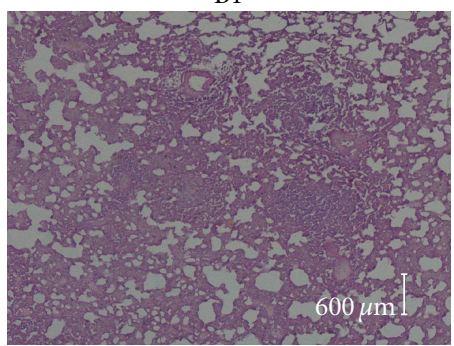

C1

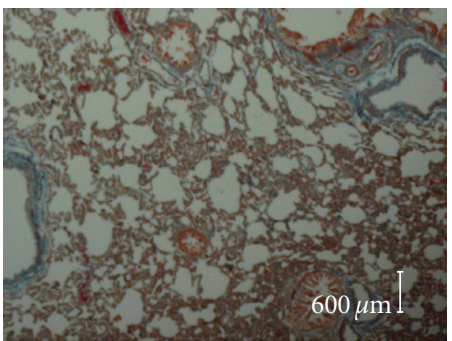

D1

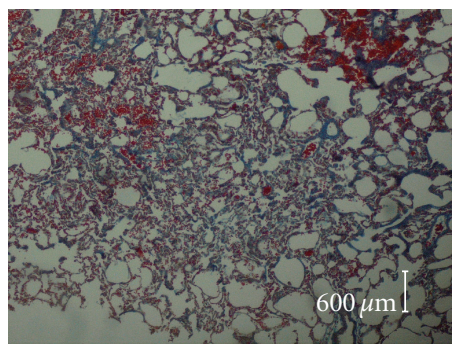

E1

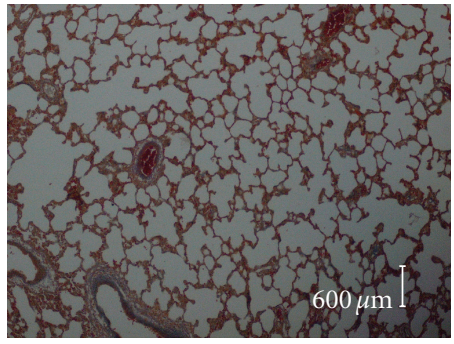

A2

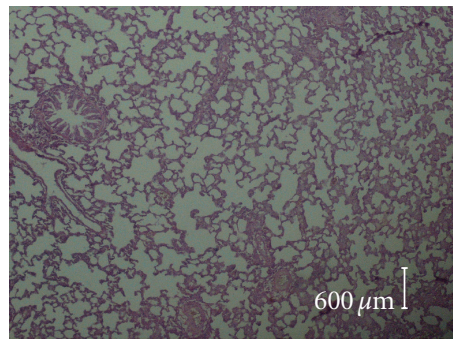

B2

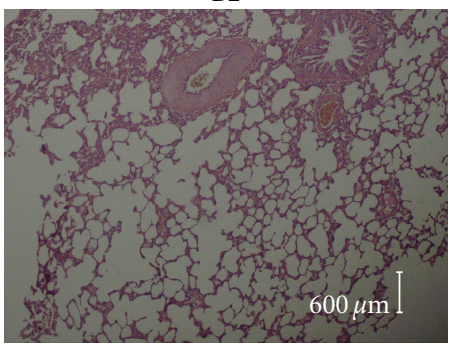

C2

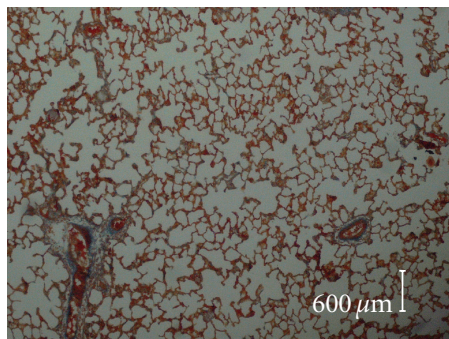

D2

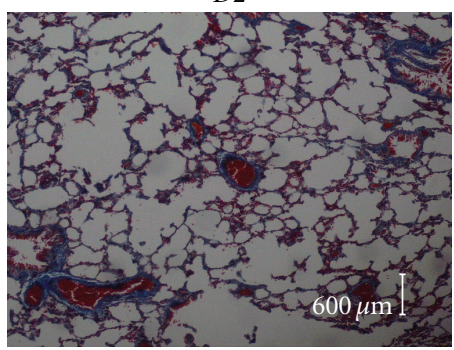

E2

FIgURE 2: The left lung histological slices of SD rats from groups of the control, PQ treatment, and PQ+PDTC treatment. In A1 (light micrographs of lung sections stained with hematoxylin and eosin (HE) from control), evidencing a normal pulmonary structure without evidence of alveolar collapse, vascular congestion, or cellular infiltrations. In B1(PQ group on the 3rd day HE), the capillary vessel of alveolar wall dilation, congestion in lungs, hemorrhagic lung, and alveolar epithelium detached, edema, and infiltration of inflammatory cell around bronchia can be visible. In B2 (PQ+PDTC group on the 3rd day HE), a slight decrease in the alveolar space can be observed as well as the existence of several infiltrative vacuolated cells in the interstitial and in the alveolar space. In C1 (PQ group on the 7th day HE), the lungs showed an accumulation of mixed inflammatory cells in the alveolar region and confluent areas of marked interstitial thickening. In $\mathrm{C} 2$ (PQ+PDTC group on the 7th day HE), there was a slight change. In A2 (MASSON from control group), relatively little collagen accumulation can be observed in control group. In D1 (PQ group on the 14th day MASSON) and E1 (PQ group on the 21st day MASSON), Masson's Trichrome staining showed collagen accumulation, broadened alveolar wall and patchy collagen deposition within the expanded interstitium in some areas. In D2 (PQ+PDTC group on the 14th day MASSON) and E2 (PQ+PDTC group on the 21st day MASSON), a slight collagen accumulation can be visible. 


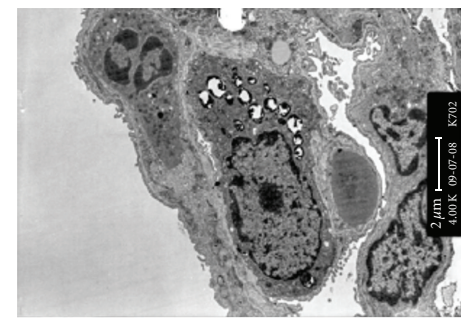

$\mathrm{A} 3$

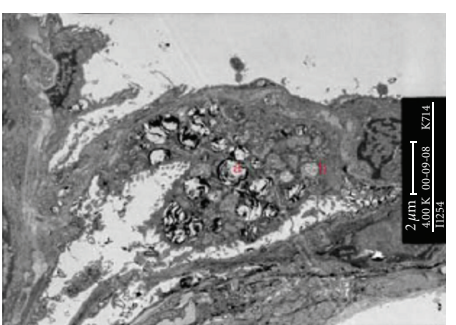

B3

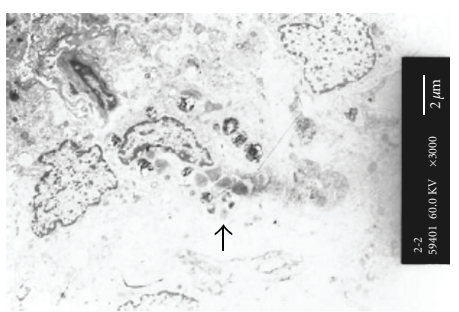

C3

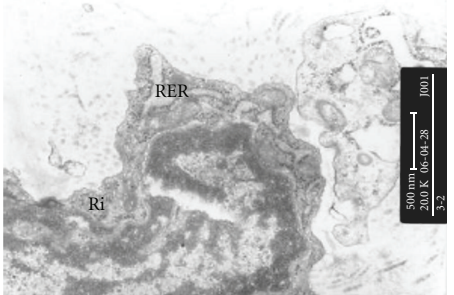

D3

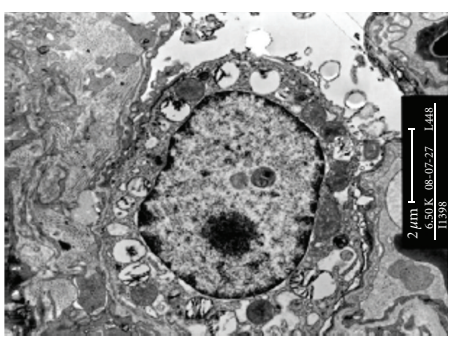

E3

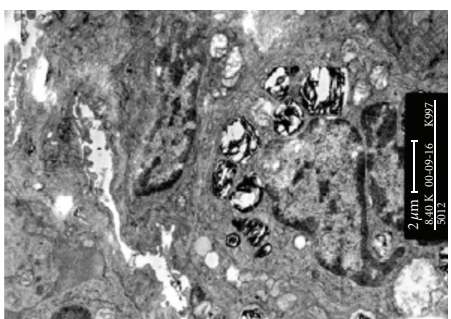

B4

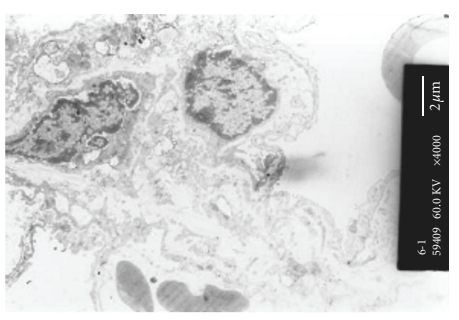

C4

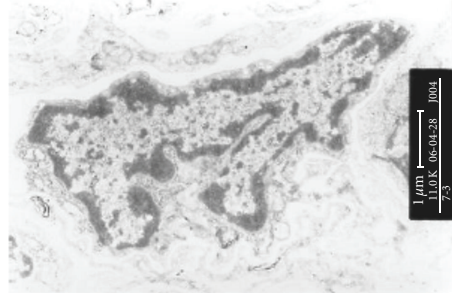

D4

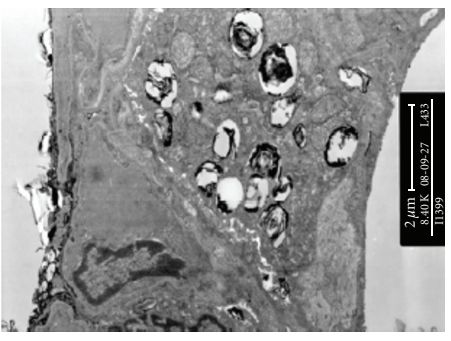

E4

FIGURE 3: The lung ultrastructure of SD rats from groups of the control, PQ treatment, and PQ+PDTC treatment. Electron micrographs from control group (A3) showed a normal ultrastructure with the presence of pneumocytes type I and II. B3 (PQ group on the 3rd day) and C3 (PQ group on the 7th day) can be observed a type II pneumocyte with mitochondrial swelling (b), dilation of lamellar body (a), pneumocyte necrosis and alveolar collapse $(\uparrow)$. B4 (PQ+PDTC group on the 3rd day) and C4 (PQ+PDTC group on the 7th day) can be observed normal mitochondria and no alveolar collapse. D3 (PQ group on the 14th day) can be noticed the more numerous rough endroplasmic reticulum (RER) and ribosome (Ri) in fibroblast compared with D4 (PQ+PDTC group on the 14th day). showed E3 (PQ group on the 21st day) can be observed patchy collagen deposition within the expanded interstitium in some areas. E4 (PQ+PDTC group on the 21st day) can be observed a small quantity of collagen deposition. 
TABLE 2: Semiquantitative analysis of the morphological injury of lung after PQ or/and PDTC treatment.

\begin{tabular}{|c|c|c|c|c|c|c|c|}
\hline Animal group & Sacrifice time $(d)$ & edema & Hyperemia & hemorrhage & inflammation & $\begin{array}{c}\text { Alveolar } \\
\text { walls thicken }\end{array}$ & $\begin{array}{l}\text { Accumulation of } \\
\text { collagenous fober }\end{array}$ \\
\hline Control & & - & - & - & - & - & - \\
\hline \multirow{4}{*}{ PQ treatment } & 3 & + & + & ++ & ++ & ++ & - \\
\hline & 7 & + & + & +++ & + & ++ & - \\
\hline & 14 & - & - & ++ & - & ++ & ++ \\
\hline & 21 & - & - & + & - & ++ & +++ \\
\hline $\mathrm{PQ}+\mathrm{PDTC}$ & 3 & - & + & + & - & - & - \\
\hline \multirow[t]{3}{*}{ treatment } & 7 & + & - & + & - & + & - \\
\hline & 14 & - & - & + & - & + & + \\
\hline & 21 & - & - & + & - & + & ++ \\
\hline
\end{tabular}

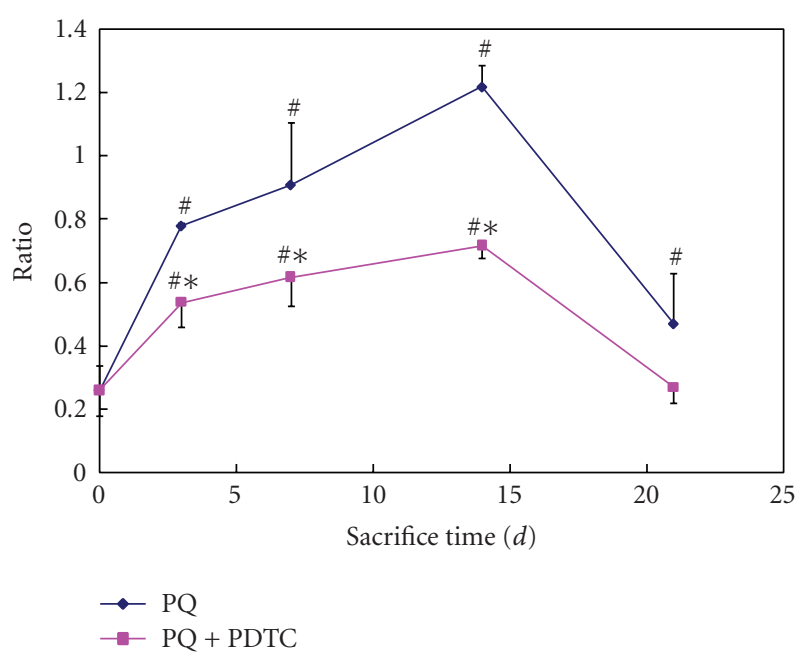

FIgURE 4: Expression TGF- $\beta 1$ mRNA in lung tissue from groups of the control, PQ treatment, and PQ + PDTC treatment. Relative TGF- $\beta 1$ mRNA abundance was normalized with the $\beta$-actin, and expressed as relative units. Values are given as mean \pm SE $(n=3)$. \# Compared with the control, $P<.05$; * compared with the PQ treatment, $P<.05$.

of the above, referred alterations were drastically attenuated in the PQ+PDTC groups, particularly inflammation, hemorrhage, and the amount of accumulation of collagenous fiber. Despite the existence of several pneumocytes with mitochondrial swelling and evidences of interstitial edema, the exuberance of those signals and the ratio of affected cells were drastically attenuated in PQ+PDTC animals. Furthermore, comparing to the PQ group, the vascular congestion and the alveolar collapse were not as noticeable in PQ+PDTC animals.

3.4. Antagonistic Effects of PDTC on the PQ-Induced Expression of TGF- $\beta 1$ Gene Expression. In order to analyze the effects of PQ on the TGF- $\beta 1$, we tested the mRNA and protein levels of TGF- $\beta 1$ in lung tissues from rats treated with PQ. As expected, the levels of both mRNA and protein of TGF- $\beta 1$ were significantly drastically increased by the PQ $(P<.05$ or $P<.01)$. Cotreatment of PDTC with PQ

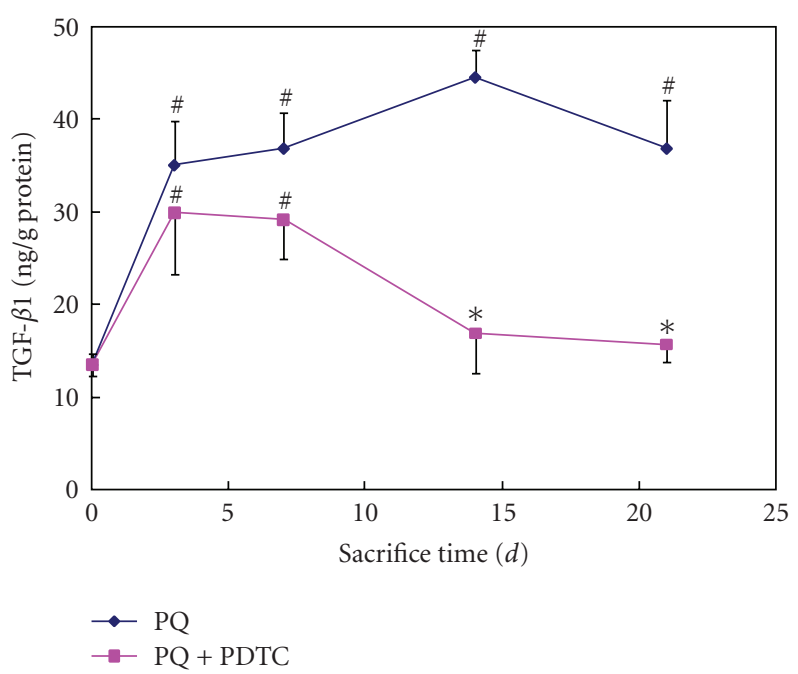

FIGURE 5: TGF- $\beta 1$ level in plasm at sacrifice time from groups of the control, PQ treatment, and PQ+PDTC treatment. TGF- $\beta 1$ was expressed as $\mathrm{ng} / \mathrm{g}$ of protein. Values are given as mean $\pm \mathrm{SE}(n=3)$. \# Compared with the control, $P<.05$; * compared with the PQ treatment, $P<.05$.

significantly decreased the levels of both mRNA and protein in comparison with PQ treatment alone $(P<.05$ or $P<.01)$ (Figures 4 and 5).

\section{Discussion}

Paraquat is a highly toxic compound for humans and animals. Over the past few decades, many cases of acute poisoning and death have been reported [22, 23]. The major cause of death in paraquat poisoning is respiratory failure due to an oxidative insult to the alveolar epithelium with subsequent obliterating fibrosis. The cellular damage mediated by PQ is essentially due to its redox-cycle leading to continuous superoxide radicals $\left(\mathrm{O}_{2}^{--}\right)$production [24]. This then sets off the well-known cascade leading to generation of the hydroxyl radical $(\mathrm{HO} \bullet)$, which has been implicated in the initiation of membrane injury by lipid peroxidation during the exposure to PQ [24-26]. In addition, researchers have 
proposed the hypothesis of cytotoxicity via mitochondrial dysfunction caused by PQ $[24,25]$. The data presented in Table 2 showed significant changes in endogenous antioxidant system and lipid peroxidation during the treatment of rats with $\mathrm{PQ}$, where the SOD and GSH-Px activities were decreased in blood plasma and BALF, and the level of MDA increased which is indicative for excessive lipid peroxidation. The aforementioned observation confirms the consequence of the intracellular accumulation of reactive oxygen species (ROS) with subsequent development of lungs injury [24, 26].

In the present study, we performed qualitative and semiquantitative analysis of the morphological injury of lung by PQ (Table 2 and Figures 2 and 3). We have observed the characteristic $\mathrm{PQ}$-induced pathological alterations including alveolar edema, hemorrhage, inflammatory cell infiltration, the swollen-type II alveolar epithelial cells, and deformed mitochondria by electro-microscopy in less than 7 days after PQ exposure. The abundant rough endoplasmic reticulum (RER) and rich ribosome in the fibroblast and the development of an extensive fibrosis in lung during 1421 days after PQ exposure, are probably a compensatory repair mechanism to damaged alveolar epithelial [27]. The alveolar walls were thickened, predominantly with collagen; mild mononuclear cell infiltration in the alveolar walls and alveolar collapse were observed in necropsy specimens of two patients 8 and 10 days after paraquat poisoning [28].

In this study, cotreatment with PDTC was very effective in the preventing oxidative damage induced by $\mathrm{PQ}$, which are characterized by the reversal of PQ-induced tissue damages. In addition, the PQ-induced biochemical changes as indicated by significant decrease of SOD and GSH-Px activities, along with an increase MDA level in blood plasm and BALF, were also alleviated by PDTC (Table 1). The mechanism of PDTC's antioxidant effect could be explained in part by activating gene expression of endogenous antioxidants such as superoxide dismutase [17] and a reduction in the accumulation of malondialdehyde [19]. In addition, inflammation was alleviated from pathological morphological analysis. These results showed that the PDTC as an antioxidant compound to counteract the toxic effects of free radicals [15] and to interfere with the generation of proinflammatory cytokines [16] efficiently protect lung from PQ-induced oxidative damage. Moreover, these morphological evidences of cellular aggression were attenuated by PDTC treatment (Table 2 and Figure 2), and the reduced accumulation of collagenous fiber observed in PQ+PDTC-treated animals during 1421 days may be interpreted as a consequence of regulated collagen gene expression, which PQ+PDTC-treated groups could attenuate paraquat-induced upregulation of TGF- $\beta 1$ mRNA expression levels.

TGF- $\beta 1$ is a key growth factor that initiates tissue repair and its sustained production underlies the development of tissue fibrosis [29]. In experimental models of lung fibrosis, TGF- $\beta 1$ has been shown to be an important upstream effector of collagen gene expression [30,31]. The attenuation of PQ-induced damages by PDTC suggests that the alleviation of PQ-induced fibrosis may be due to the inhibitory effects of PDTC on NF- $\kappa$ B activation which then led to reduced TGF$\beta 1$ gene expression [32].
In conclusion, our results in the present study clearly demonstrated that PDTC significantly increased SOD, GSHPx activities, decreased MDA, HYP levels, and reduced accumulation of collagenous fiber in paraquat-treated rat. These findings suggested that PDTC may exert its protective effects on paraquat-induced pulmonary damage by alleviating the earlier inflammation damage via paraquat-induced oxidative stress and the later fibrosis in rat lung and by regulating the mRNA expression of TGF- $\beta 1$. Future studies are warranted to further investigate the underlying mechanisms involved in this complicated process.

\section{Acknowledgments}

This research was supported by the Chinese Ministry of Science and Technology (2006BA106B01) and Special Fund of Shanghai Health Bureau (08GWZX0303,08GWD20). We thank Dr. Yanan Tian in the Department of Veterinary Physiology and Pharmacology of Texas A \& M University for helpful comments on this manuscript.

\section{References}

[1] R. J. Dinis-Oliveira, M. J. De Jesús Valle, M. L. Bastos, F. Carvalho, and A. Sánchez Navarro, "Kinetics of paraquat in the isolated rat lung: influence of sodium depletion," Xenobiotica, vol. 36, no. 8, pp. 724-737, 2006.

[2] R. J. Dinis-Oliveira, F. Remião, H. Carmo, et al., "Paraquat exposure as an etiological factor of Parkinson's disease," NeuroToxicology, vol. 27, no. 6, pp. 1110-1122, 2006.

[3] C. P. Lewis and B. Nemery, Pathophysiology and Biochemical Mechanisms of the Pulmonary Toxicity of Paraquat, vol. 10, Marcel Dekker, New York, NY, USA, 1995.

[4] H. W. Gil, M. H. Oh, K. M. Woo, E. Y. Lee, M. H. Oh, and S. Y. Hong, "Relationship between pulmonary surfactant protein and lipid peroxidation in lung injury due to paraquat intoxication in rats," The Korean Journal of Internal Medicine, vol. 22, no. 2, pp. 67-72, 2007.

[5] M. Yamashita, M. Yamashita, and Y. Ando, "A long-term follow-up of lung function in survivors of paraquat poisoning," Human and Experimental Toxicology, vol. 19, no. 2, pp. 99-103, 2000.

[6] D. N. Bateman, "Pharmacological treatments of paraquat poisoning," Human Toxicology, vol. 6, no. 1, pp. 57-62, 1987.

[7] P. Honore, P. Hantson, J. P. Fauville, A. Peeters, and P. Mahieu, "Paraquat poisoning," Acta Clinica Belgica, vol. 49, no. 5, pp. 220-228, 1994.

[8] A. T. Proudfoot, L. F. Prescott, and D. R. Jarvie, "Haemodialysis for paraquat poisoning," Human Toxicology, vol. 6, no. 1, pp. 69-74, 1987.

[9] Z. E. Suntres, "Role of antioxidants in paraquat toxicity," Toxicology, vol. 180, no. 1, pp. 65-77, 2002.

[10] C. Li, W. Browder, and R. L. Kao, "Early activation of transcription factor NF- $\kappa \mathrm{B}$ during ischemia in perfused rat heart," American Journal of Physiology, vol. 276, no. 2, pp. H543-H552, 1999.

[11] S. Orrenius, C. S. I. Nobel, D. J. van den Dobbelsteen, M. J. Burkitt, and A. F. G. Slater, "Dithiocarbamates and the redox regulation of cell death," Biochemical Society Transactions, vol. 24, no. 4, pp. 1032-1038, 1996. 
[12] A. Iseki, F. Kambe, K. Okumura, et al., "Pyrrolidine dithiocarbamate inhibits TNF- $\alpha$-dependent activation of NF- $\kappa \mathrm{B}$ by increasing intracellular copper level in human aortic smooth muscle cells," Biochemical and Biophysical Research Communications, vol. 276, no. 1, pp. 88-92, 2000.

[13] S. D. Ross, I. L. Kron, J. J. Gangemi, et al., "Attenuation of lung reperfusion injury after transplantation using an inhibitor of nuclear factor- $\kappa \mathrm{B}$," American Journal of Physiology, vol. 279, no. 3, pp. L528-L536, 2000.

[14] R. Schreck, B. Meier, D. N. Männel, W. Dröge, and P. A. Baeuerle, "Dithiocarbamates as potent inhibitors of nuclear factor $\kappa \mathrm{B}$ activation in intact cells," Journal of Experimental Medicine, vol. 175, no. 5, pp. 1181-1194, 1992.

[15] D. N. Muller, R. Dechend, E. M. A. Mervaala, et al., "NF- $\kappa B$ inhibition ameliorates angiotensin II-induced inflammatory damage in rats," Hypertension, vol. 35, no. 1, part 2, pp. 193201, 2000.

[16] S. F. Liu, X. Ye, and A. B. Malik, "Inhibition of NF- $\kappa$ B activation by pyrrolidine dithiocarbamate prevents in vivo expression of proinflammatory genes," Circulation, vol. 100, no. 12, pp. 1330-1337, 1999.

[17] S. Borrello and B. Demple, "NFאB-independent transcriptional induction of the human manganous superoxide dismutase gene," Archives of Biochemistry and Biophysics, vol. 348, no. 2, pp. 289-294, 1997.

[18] A. C. Wild and R. T. Mulcahy, "Pyrrolidine dithiocarbamate up-regulates the expression of the genes encoding the catalytic and regulatory subunits of $\gamma$-glutamylcysteine synthetase and increases intracellular glutathione levels," Biochemical Journal, vol. 338, no. 3, pp. 659-665, 1999.

[19] A. B. Nathens, R. Bitar, C. Davreux, et al., "Pyrrolidine dithiocarbamate attenuates endotoxin-induced acute lung injury," American Journal of Respiratory Cell and Molecular Biology, vol. 17, no. 5, pp. 608-616, 1997.

[20] S. V. Szapiel, N. A. Elson, J. D. Fulmer, G. W. Hunninghake, and R. G. Crystal, "Bleomycin-induced interstitial pulmonary disease in the nude, athymic mouse," American Review of Respiratory Disease, vol. 120, no. 4, pp. 893-899, 1979.

[21] O. H. Lowry, N. J. Rosebrough, A. L. Farr, and R. J. Randall, "Protein measurement with the Folin phenol reagent," The Journal of Biological Chemistry, vol. 193, no. 1, pp. 265-275, 1951.

[22] A. Autor, Biochemical Mechanisms of Paraquat Toxicity, Academic Press, New York, NY, USA, 1977.

[23] T. E. Gram, "Chemically reactive intermediates and pulmonary xenobiotic therapy," Pharmacological Reviews, vol. 49, no. 4, pp. 297-341, 1997.

[24] J. S. Bus, S. D. Aust, and J. E. Gibson, "Superoxide and singlet oxygen catalyzed lipid peroxidation as a possible mechanism for paraquat (methyl viologen) toxicity," Biochemical and Biophysical Research Communications, vol. 58, no. 3, pp. 749 755, 1974.

[25] C.-M. Chen and A. C. Lua, "Lung toxicity of paraquat in the rat," Journal of Toxicology and Environmental Health Part A, vol. 60 , no. 7, pp. 477-487, 2000.

[26] R. J. Youngman and E. F. Elstner, "Oxygen species in paraquat toxicity: the crypto-OH radical," FEBS Letters, vol. 129, no. 2, pp. 265-268, 1981.

[27] P. Smith and D. Heath, "Paraquat," Critical Reviews in Toxicology, vol. 4, no. 4, pp. 411-445, 1976.

[28] S. H. Lee, K. S. Lee, J. M. Ahn, S. H. Kim, and S. Y. Hong, "Paraquat poisoning of the lung: thin-section CT findings," Radiology, vol. 195, no. 1, pp. 271-274, 1995.
[29] W. A. Border and E. Ruoslahti, "Transforming growth factor$\beta$ in disease: the dark side of tissue repair," Journal of Clinical Investigation, vol. 90, no. 1, pp. 1-7, 1992.

[30] J. Gauldie, P. J. Sime, Z. Xing, B. Marr, and G. M. Tremblay, "Transforming growth factor-beta gene transfer to the lung induces myofibroblast presence and pulmonary fibrosis," Current Topics in Pathology, vol. 93, pp. 35-45, 1999.

[31] N. J. Kenyon, R. W. Ward, G. McGrew, and J. A. Last, "TGF- $\beta 1$ causes airway fibrosis and increased collagen I and III mRNA in mice," Thorax, vol. 58, no. 9, pp. 772-777, 2003.

[32] Y.-C. Fong, M.-C. Maa, F.-J. Tsai, et al., "Osteoblast-derived TGF- $\beta 1$ stimulates IL- 8 release through AP- 1 and NF- $\kappa$ B in human cancer cells," Journal of Bone and Mineral Research, vol. 23, no. 6, pp. 961-970, 2008. 


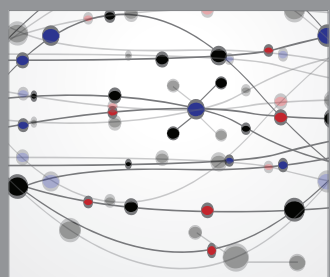

The Scientific World Journal
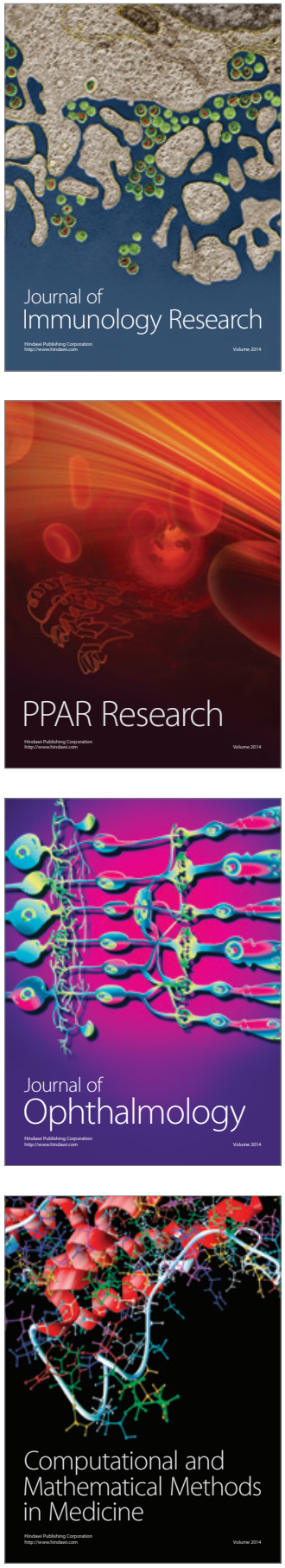

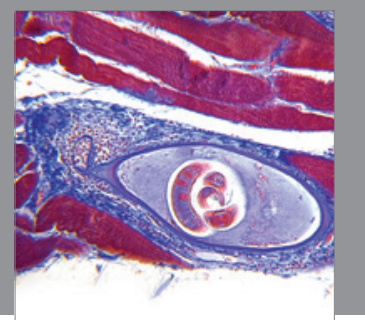

Gastroenterology

Research and Practice
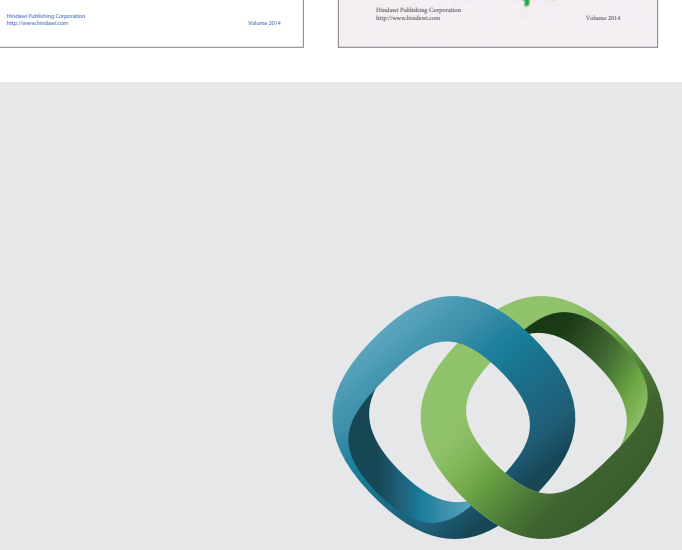

\section{Hindawi}

Submit your manuscripts at

http://www.hindawi.com
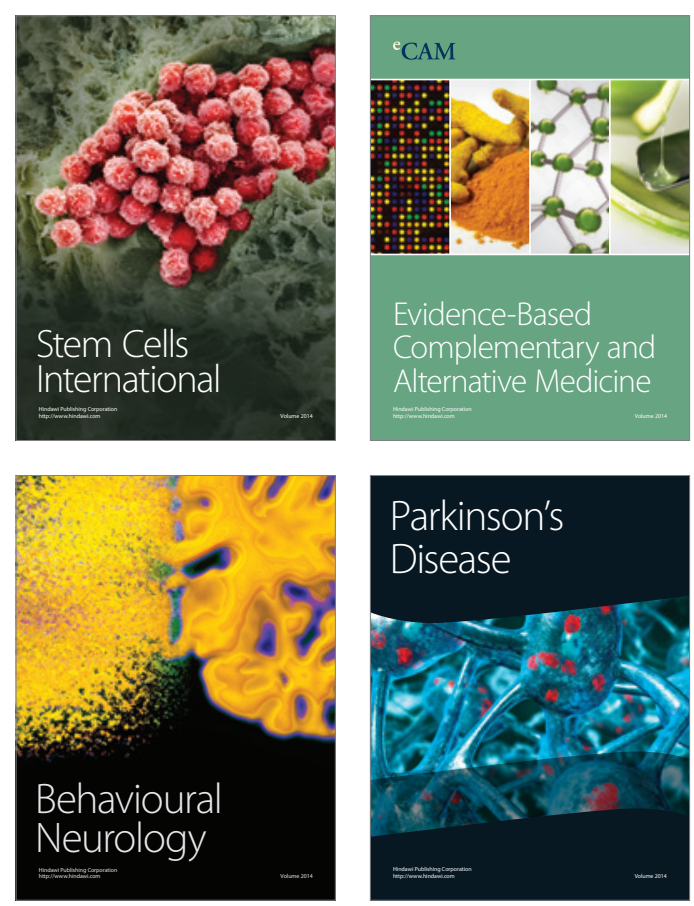

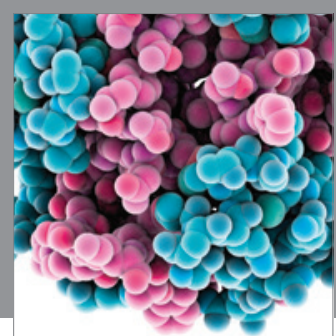

Journal of
Diabetes Research

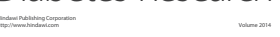

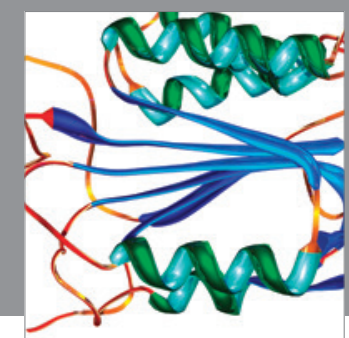

Disease Markers
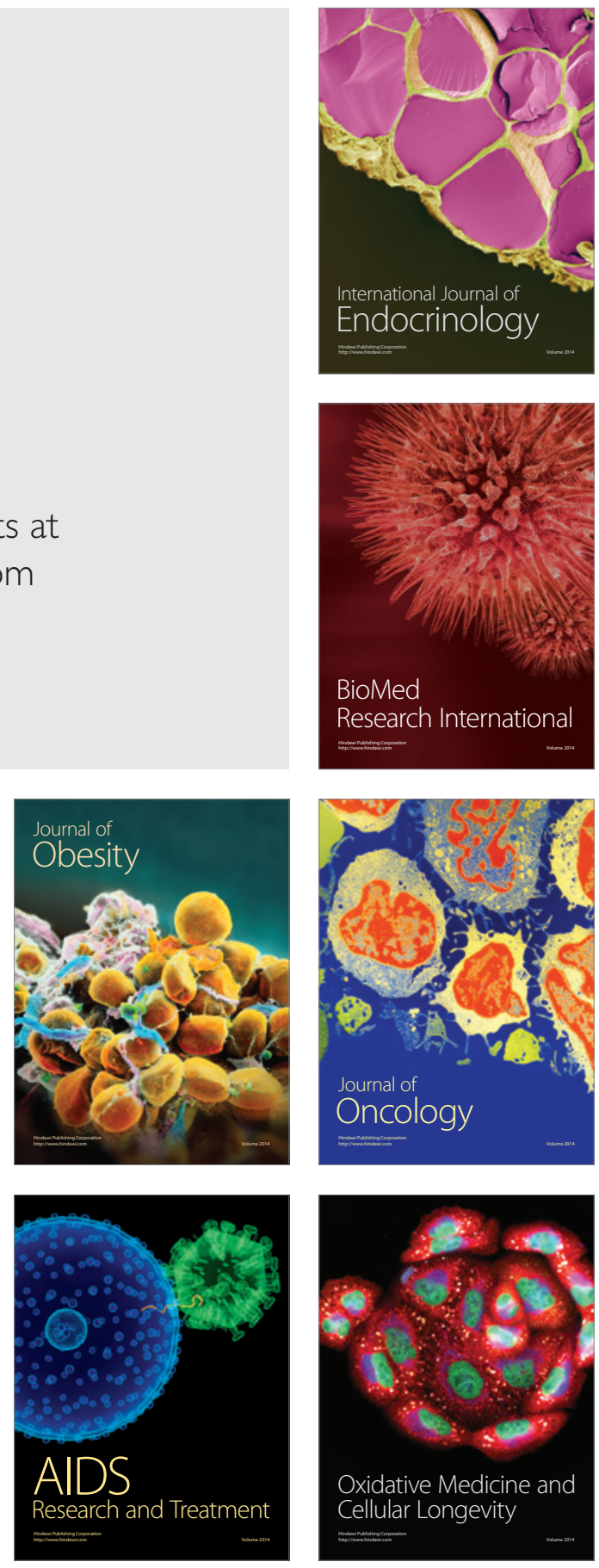